DOI: $10.3901 / J M E .2019 .18 .036$

\title{
复合结构衬套合金界面结合性能*
}

\author{
王建梅 夏全志 侯定邦 姚 坤 \\ (太原科技大学重型机械教育部工程研究中心 太原 030024)
}

\begin{abstract}
摘要: 为了深入探讨层状金属复合材料连接界面的结合性能, 以新型复合结构祄套巴氏合金 ZChSnSb8-4 为研究对象, 在原 子替代法建模特点的基础上，考虑巴氏合金中 $\mathrm{Cu}_{6} \mathrm{Sn}_{5} 、 \mathrm{SnSb} 、 \mathrm{Sn}$ 三种组分含量配比，对多层合金复合祄套界面间结合性能 进行分子动力学模拟分析。计算结果表明: 结合界面的层数对结合性能有着明显的影响, 五层复合结构祄套的最小界面结合 能比三层复合结构祄套的最小界面结合能大 $33.87 \%$, 即五层复合结构祄套界面结合性能优于三层复合结构祄套。同时从不 同结构祄套相邻两界面的结合能发现，不同复合层结构的祄套可能导致的危险结合界面不同。钢铅合金层与镍㮽层之间的界 面结合能最大, 结合最牢固; 镍栅层与巴氏合金层的界面结合能最小, 最易发生合金脱落现象。从分子层面研究了新型复合 结构祄套界面的结合机理，可为生产实践提供参考依据。
\end{abstract}

关键词: 分子动力学; 复合材料; 巴氏合金; 界面结合能; 结合机理

中图分类号: TH117

\section{Interfacial Bonding Properties of Metal Bushing with Composite Structure}

\author{
WANG Jianmei XIA Quanzhi HOU Dingbang YAO Kun
}

(Engineering Research Center of Heavy Machinery, Ministry of Education, Taiyuan University of Science and Technology, Taiyuan 030024)

\begin{abstract}
In order to investigate the bonding properties of the interface of laminated metal composites, a new type of composite structure bushing babbitt alloy ZChSnSb8-4 is studied based on the modelling characteristics of atomic substitution method. Considering the proportion of three components of $\mathrm{Cu}_{6} \mathrm{Sn}_{5}, \mathrm{SnSb}, \mathrm{Sn}$ in babbitt alloy, the interfacial bonding properties of multilayer alloy composite bushing are simulated and studied using molecular dynamics method. The results show that the minimum interfacial bonding energy of five-layer structure bushing is $33.87 \%$ higher than that of three-layer structure bushing, so the interfacial bonding performance of five-layer structure bushing is better than that of three-layer structure bushing. Meanwhile, according to the bonding energy of the adjacent two interfaces of different structure bushing, it is found that the dangerous bonding interface may be caused by different composite layer bushing. The interfacial bonding energy betweensteel-lead alloy layer andnickel gate layer is the largest and the bond is the strongest, and the interface bonding energy between nickel gate layer and babbitt alloy layer is the least, and the phenomenon of alloy falling off is the most likely. A new type of compound structure bushing is studied from the molecular level, and the bonding mechanism of the interfaces provides a reference for the production practice.
\end{abstract}

Key words: molecular dynamics; composite; babbitt; interface bonding energy; bonding mechanism

\section{0 前言}

随着现代科学技术发展, 单一的金属或合金已 经很难完全满足现代化的工业生产对材料的综合性 能的需要, 以层状结合而形成的异种金属新型复合 材料, 近年来受到世界各国的普遍重视。层状金属 在保持母材金属特性的同时具有 “相补效应”, 可以

* 国家自然科学基金(51875382, U1610109)、山西省重点研发计划(指 南)(201803D421103)和太原重型机械装备协同创新中心专项(1331 工程) 资助项目。20181011 收到初稿, 20190222 收到修改稿
弥补各自的不足 ${ }^{[1]}$ 。经过恰当的组合易于形成优异 的综合性能, 可改善材料的力学性能、热电性能、 耐腐蚀性等诸多性能 ${ }^{[2]}$ 。其中, 油膜轴承祄套就属 于典型的层状金属复合材料 ${ }^{[3]}$ 。油膜轴承由于其摩 擦因数小、损耗低、刚性高等优点, 广泛应用于钢 铁、矿山、冶金、电力等系统的高、精、尖关键设 备 ${ }^{[4]}$ 。祄套作为油膜轴承的核心部件, 其结构和运 行过程中的受力情况对油膜轴承最终的使用性能起 着至关重要的作用 ${ }^{[5]}$ 。其各层合金间的界面结合性 能关系到油膜轴承最终的使用性能, 影响着生产线 的安全可靠性 ${ }^{[6-7]}$ 。轴承祄套由硬质基体金属与较软 
金属相结合组成, 在承受重载和较大的冲击载荷下, 其内壁巴氏合金极易因结合性能不够而导致脱落损 坏导致轴承失效。工程应用中一旦出现此类情况, 必然导致生产线停产, 严重影响生产进度, 造成重 大经济损失, 甚至会危及生产人员的生命安全。因 此, 轴承祄套多层合金界面结合性能的好坏直接关 系到大型设备的使用安全, 对多层合金制备工艺和 发展新型结合技术具有重要的理论指导意义。研究 新型油膜轴承多层祄套界面结合性能对设备结构与 运行至关重要 ${ }^{[8-10]}$ 。

分子动力学方法能够在原子或分子等纳尺度上 进行建模计算, 已经广泛应用于复合材料界面的模 拟计算中。LUO 等 ${ }^{[11]}$ 在微观上研究 Mo-Ti 界面的扩 散键合对连接复合材料结构内在关联, 并对界面进 行了精细分布和 Z 轴浓度分布的表征。CHEN 等 ${ }^{[12]}$ 应用分子动力学对铂表面超薄液氩膜的气泡成核现 象进行了研究。BEJAUD 等 ${ }^{[13]}$ 研究了纳米 $\mathrm{Cu} / \mathrm{Ag}$ 中 形变孪晶与界面的相互作用。ADJAOUD 等 ${ }^{[14]}$ 采用 分子动力学模拟了 $\mathrm{Pd} 80 \mathrm{Si} 20$ 和 Cu64Zr36 纳米玻璃 球的微观结构形成过程。WU 等 ${ }^{[15]}$ 采用基于多体嵌 入原子势的分子动力学模拟方法, 研究了模腔几何 形状和压印温度对印迹非晶 $\mathrm{ZrCu}$ 薄膜的影响。

LASHGARI 等 ${ }^{[16]}$ 从纳米角度研究了 $\mathrm{Fe}-\mathrm{Si}$ 合金循环 压痕导致的应力松弛和应变硬化行为。MOJUMDER 等 ${ }^{[17]}$ 在分子层面研究了载荷作用对 $\mathrm{Al}-\mathrm{Cu}$ 合金不同 $\mathrm{Al}$ 晶体取向与合金元素的可塑性 $(\mathrm{Cu})$ 比例关系, 解 释了纳米合金的压应力应变行为。SARKAR 等 ${ }^{[18]}$ 研究了单层、矩形、平面和原始硅烯片在纳米缩进 过程中的力学行为和响应过程。分子动力学的发展 给新型多层结构轴承祄套合金间的界面结合性能问 题提供了新的研究思路。

为了深入探讨层状金属复合材料连接界面的 结合性能, 以新型油膜轴承复合祄套巴氏合金 ZChSnSb8-4 为研究对象, 通过分析祄套合金各层材 料的组分, 计算其各组分含量比, 然后根据其组分 比运用分子动力学方法进行建模, 对其合金界面结 合能进行模拟, 对比分析三层与五层复合结构祄套 结合性能的关系, 并对每相邻合金所组成界面的结 合能进行计算, 给出复合材料危险界面的判定依据, 从纳观层面分析了复合材料界面结合的机理。

\section{1 基于材料组分的多层合金界面模拟}

\section{1 模型的构建}

油膜轴承复合祄套, 三层复合结构和五层复合 结构如图 1 所示。构建模型不同条件下的界面结构
时, 需要查询晶体结构的一些参数, 包括晶体的空 间群、晶格参数和晶体内原子的坐标等。查询无机 晶体结构数据库(ICSD), 文中所需要构建的材料模 型相关参数如表 1 所示。

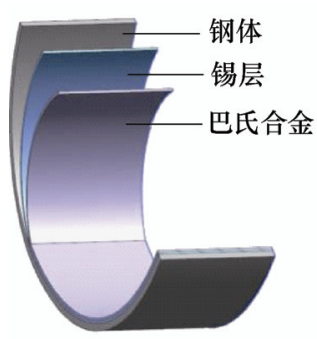

(a) 三层复合结构祄套

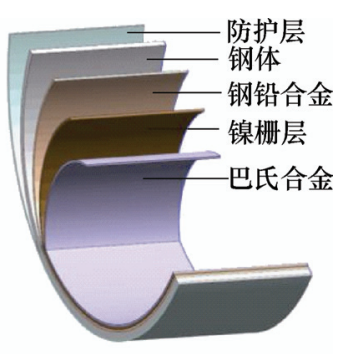

(b) 五层复合结构祄套
图 1 不同复合结构衬套

表 1 材料模型晶胞结构参数

\begin{tabular}{cccccc}
\hline 材料 & 钢体 & 防护层 & 钢铅合金 & 镍桶层 \\
\hline 空间群 & CMC21 & FD-3M & I42-M & FM-3M \\
\hline 晶系 & & 正交晶系 & 正交晶系 & 正交晶系 & 正交晶系 \\
\hline \multirow{2}{*}{ 晶胞长度/ } & $a$ & 10.108 & 6.491 & 5.460 & 5.964 \\
$\times 10^{-10} \mathrm{~m}$ & $b$ & 7.998 & 6.491 & 5.460 & 5.964 \\
& $c$ & 7.546 & 6.491 & 10.725 & 5.964 \\
\hline \multirow{2}{*}{ 晶胞角度/ } & $\alpha$ & 90 & 90 & 90 & 90 \\
$\left({ }^{\circ}\right)$ & $\beta$ & 90 & 90 & 90 & 90 \\
& $\gamma$ & 90 & 90 & 90 & 90 \\
\hline
\end{tabular}

利用软件中的 Build crystals 工具 ${ }^{[19]}$, 根据材料 晶胞的参数进行晶胞的构建, 分别构建好所需防护 层、钢体、钢铅合金、镍栅层材料的原晶胞结构。 锡的原晶胞结构可以从软件的材料库中直接导入。 构建好晶胞结构如图 2 所示。

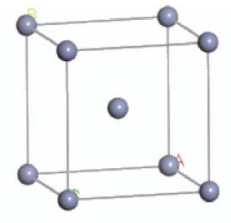

(a) 防护层晶胞结构

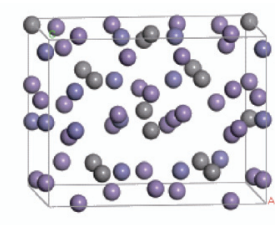

(b) 钢体晶胞结构

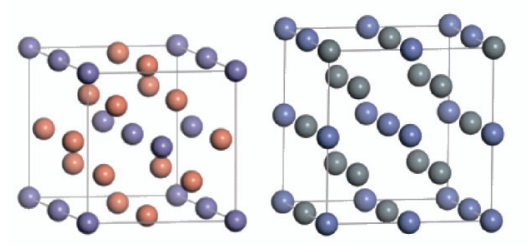

(c) 钢铅合金晶胞结构

(d) 镍栅层晶胞结构

图 2 材料原晶胞结构

接着, 需要对于构建好的晶胞结构进行切面, 切面选择的一般原则是对低指数面即界面能最低的 面进行剪切。利用 Cleave Surface 工具对于各晶胞 进行简单切面。为了在构建界面时尺寸相近, 不至 于产生较大的误差, 切面之后将所有晶胞结构扩展 
为超晶胞, 利用 Build Vacuum Slab 工具将构建好的 超晶胞结构分别从 $2 \mathrm{D}$ 结构改为 $3 \mathrm{D}$ 结构, 并选择合 适的真空层厚度。

对于巴氏合金的晶胞结构，其主要由 $\mathrm{Cu}_{6} \mathrm{Sn}_{5}$ 、 $\mathrm{SnSb} 、 \mathrm{Sn}$ 三种成分组成，以锡基巴氏合金 $\mathrm{ZChSnSb} 8-4$ 为例。其中 $\mathrm{Cu}$ 含量为 $4 \%, \mathrm{Sb}$ 含量为 $8 \%$, 其余含量为 $\mathrm{Sn}$ 。根据各原子的相对原子质量, 则下式成立

$$
\left\{\begin{array}{l}
m_{\mathrm{Cu}} \cdot 6 n_{\mathrm{Cu}_{6} \mathrm{Sn}_{5}}=M_{\mathrm{T}} \cdot \omega_{\mathrm{Cu}} \\
m_{\mathrm{Sb}} \cdot n_{\mathrm{Snsb}}=M_{\mathrm{T}} \cdot \omega_{\mathrm{Sb}} \\
m_{\mathrm{Sn}} \cdot\left(5 n_{\mathrm{Cu}_{6} \mathrm{Sn}_{5}}+n_{\mathrm{SnSb}}+n_{\mathrm{Sn}}\right)=M_{\mathrm{T}} \cdot \omega_{\mathrm{Sn}}
\end{array}\right.
$$

式中, $m_{\mathrm{Cu}} 、 m_{\mathrm{Sb}} 、 m_{\mathrm{Sn}}$ 分别为 $\mathrm{Cu} 、 \mathrm{Sb} 、 \mathrm{Sn}$ 的相对原 子质量, $n_{\mathrm{Cu}_{6} \mathrm{Sn}_{5}} 、 n_{\mathrm{SnSb}} 、 n_{\mathrm{Sn}}$ 分别为 $\mathrm{Cu}_{6} \mathrm{Sn}_{5} 、 \mathrm{SnSb}$ 、 $\mathrm{Sn}$ 物质的量, $\omega_{\mathrm{Cu}} 、 \omega_{\mathrm{Sb}} 、 \omega_{\mathrm{Sn}}$ 分别为 $\mathrm{Cu} 、 \mathrm{Sb} 、 \mathrm{Sn}$ 的

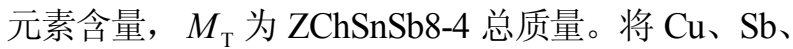
$\mathrm{Sn}$ 的相对原子质量代入上式中得到 $\mathrm{Cu}_{6} \mathrm{Sn}_{5} 、 \mathrm{SnSb} 、 \mathrm{Sn}$ 三种成分的含量比为 $1: 6: 60$ 。则可根据三种组分的 含量之比构建巴氏合金的超晶胞结构, 如图 3 所示。

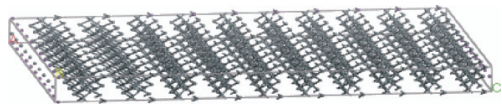

图 3 巴氏合金超晶胞结构

对构建好的所有材料的超晶胞结构进行力场分 配, 选用软件内置的 COMPASS 力场 ${ }^{[20]}$, 在 Typing 功能下对晶胞中所有原子赋予力场。优化时同时考虑 范德华力和库仑力, 用 Minimizer 功能对分配好力场 的晶胞结构表面进行能量最小化, 从而优化表面结 构, 使得优化原子位置更加精确 ${ }^{[21]}$ 。优化之前需固定 晶胞结构底部的原子, 只对表面几层原子进行表面优 化, 以达到表面能量最小化。Minimizer 法对系统的 结构进行改善, 从而达到最稳定的构型 ${ }^{[22]}$, 使得在后 续的动力学模拟时能量波动较小, 收玫时间更短。

利用 Build layer 工具对不同条件下的界面结构 进行构建，依照顺序将表面优化后的巴氏合金层、 钢体层、锡层构建三层晶胞结构, 将保护层、钢体、 钢铅合金、镍栅层、巴氏合金层构建五层晶胞结构。 同时调整晶胞结构的方向，使经过优化后的表面相 对应, 构建好的三层与五层复合结构分子模型分别 如图 4 所示。

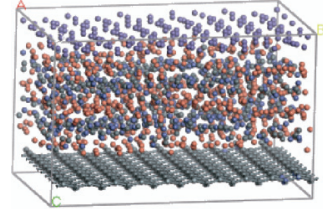

(a) 三层复合结构分子模型

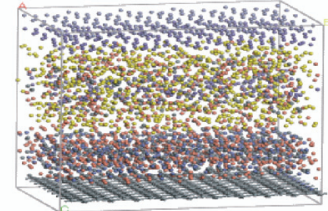

(b) 五层复合结构分子模型
图 4 不同复合结构的分子模型

\section{2 模拟方法}

所有模拟计算都在 Discover 模块中进行，优化 其表面时, 选择能量最优化计算方法。考虑运算量 和运算速度, 在动力学计算前先对构建好的界面结 构远离界面的几层原子进行固定，因为只有表面处 的少数原子会发生相互作用 ${ }^{[23]}$ 。Molecular Dynamics 中采用恒定原子数 $(\mathrm{N})$ 、恒定体积 $(\mathrm{V})$ 、恒定温度 $(\mathrm{T})$ 的正则系综(NVT), 由文献[24]得出结合能最大时的 温度为 $512 \mathrm{~K}$, 故设定初始温度为 $512 \mathrm{~K}$, 选择合适 的温度控制算法，模拟时间和时间步长，对平衡后 的结构进行能量计算。

计算相邻合金间界面结合能时，需要在界面结 构分子动力学模拟后的轨迹文件中去除多余材料, 得到相邻两层材料结合的分子动力学模型, 此时结 构已经处于平衡稳定状态，相邻两层材料结合模型 如图 5 所示。

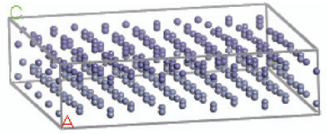

(a) 防护层与钢体层

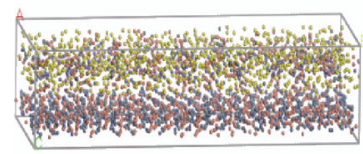

(c) 钢铅合金层与镍桶层

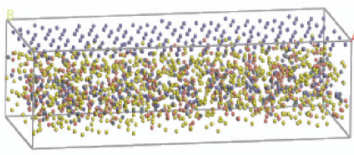

(b) 钢体层与钢铅合金层

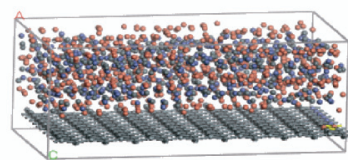

(d) 镍栅层与巴氏合金层
图 5 不同复合层间的分子结构

\section{3 界面结合能计算}

通常利用界面结合能来评价界面的结合性 能, 如果界面结合能越大, 则破坏界面平衡结构 需做的功就越多，界面结合就越牢固。由文献[25] 提出的结合能计算方法和文献[26]提出的三层材 料界面结合能计算式。定义界面结合能为两种物 质界面之间的相互作用能。计算三层材料结合能 可根据式(2)

$$
\left\{\begin{array}{l}
E_{\mathrm{I}_{1}}=E_{\mathrm{S}-\mathrm{Sn}}-\left(E_{\mathrm{S}}+E_{\mathrm{Sn}}\right) \\
E_{\mathrm{I}_{2}}=E_{\mathrm{Sn}-\mathrm{B}}-\left(E_{\mathrm{Sn}}+E_{\mathrm{B}}\right)
\end{array}\right.
$$

式中, $E_{\mathrm{I}_{1}}$ 为三层复合结构中钢体与 $\mathrm{Sn}$ 层界面结合 能, $\mathrm{kJ} \cdot \mathrm{mol}^{-1} ; E_{\mathrm{I}_{2}}$ 为三层复合结构中 $\mathrm{Sn}$ 层与巴氏 合金层界面结合能, $\mathrm{kJ} \cdot \mathrm{mol}^{-1} ; E_{\mathrm{S}-\mathrm{Sn}}$ 为钢体与 $\mathrm{Sn}$ 层 的总能量, $\mathrm{kJ} \cdot \mathrm{mol}^{-1} ; E_{\mathrm{Sn}-\mathrm{B}}$ 为 $\mathrm{Sn}$ 层与巴氏合金层的 总能量, $\mathrm{kJ} \cdot \mathrm{mol}^{-1} ; E_{\mathrm{S}}$ 为钢体层能量, $\mathrm{kJ} \cdot \mathrm{mol}^{-1} ; E_{\mathrm{Sn}}$ 为 $\mathrm{Sn}$ 层能量, $\mathrm{kJ} \cdot \mathrm{mol}^{-1} ; E_{\mathrm{B}}$ 为巴氏合金层能量, $\mathrm{kJ} \cdot \mathrm{mol}^{-1}$ 。

由以上推导式, 五层材料祄套每相邻界面结合 能如下 


$$
\left\{\begin{array}{l}
E_{\mathrm{I}_{3}}=E_{\mathrm{P}-\mathrm{S}}-\left(E_{\mathrm{P}}+E_{\mathrm{S}}\right) \\
E_{\mathrm{I}_{4}}=E_{\mathrm{S}-\mathrm{L}}-\left(E_{\mathrm{S}}+E_{\mathrm{L}}\right) \\
E_{\mathrm{I}_{5}}=E_{\mathrm{L}-\mathrm{N}}-\left(E_{\mathrm{L}}+E_{\mathrm{N}}\right) \\
E_{\mathrm{I}_{6}}=E_{\mathrm{N}-\mathrm{B}}-\left(E_{\mathrm{N}}+E_{\mathrm{B}}\right)
\end{array}\right.
$$

式中, $E_{\mathrm{I}_{3}}$ 为五层复合结构中防护层与钢体层界 面结合能, $\mathrm{kJ} \cdot \mathrm{mol}^{-1} ; E_{\mathrm{I}_{4}}$ 为五层复合结构中钢体 与钢铅合金层的界面结合能, $\mathrm{kJ} \cdot \mathrm{mol}^{-1} ; E_{\mathrm{I}_{5}}$ 为五 层复合结构中钢铅合金层与镍栅层的界面结合 能, $\mathrm{kJ} \cdot \mathrm{mol}^{-1} ; E_{\mathrm{I}_{6}}$ 为五层复合结构中镍栅层与巴 氏合金层的界面结合能, $\mathrm{kJ} \cdot \mathrm{mol}^{-1} ; E_{\mathrm{P}-\mathrm{S}}$ 为防护层 与钢体层的总能量, $\mathrm{kJ} \cdot \mathrm{mol}^{-1} ; E_{\mathrm{S}-\mathrm{L}}$ 为钢体层与 钢铅合金层的总能量, $\mathrm{kJ} \cdot \mathrm{mol}^{-1} ; E_{\mathrm{L}-\mathrm{N}}$ 为钢铅合
金层与镍栅层的总能量, $\mathrm{kJ} \cdot \mathrm{mol}^{-1} ; E_{\mathrm{N}-\mathrm{B}}$ 为镍栅 层与巴氏合金层的总能量, $\mathrm{kJ} \cdot \mathrm{mol}^{-1}$ 。 $E_{\mathrm{P}}$ 为防护 层能量, $\mathrm{kJ} \cdot \mathrm{mol}^{-1} ; E_{\mathrm{S}}$ 为钢体层能量, $\mathrm{kJ} \cdot \mathrm{mol}^{-1}$; $E_{\mathrm{L}}$ 为钢铅合金层能量, $\mathrm{kJ} \cdot \mathrm{mol}^{-1} ; E_{\mathrm{N}}$ 为镍栅层能 量, $\mathrm{kJ} \cdot \mathrm{mol}^{-1}$ 。

计算所有能量时, 需要将整个结构中原子的约 束去除。计算其中一种材料的能量时, 需要将界面 结构中的其他材料原子进行删除, 然后对其进行计 算。该计算规律可以推广用于计算其他多层材料界 面结构的结合能计算中 ${ }^{[27]}$ 。

三层和五层复合结构中总能量及其各层材料的 能量如表 2 所示。对于界面不同的能量进行分析, 各类型界面能量组成见表 3 。

表 2 不同复合结构各层材料能量

$\mathrm{kJ} \cdot \mathrm{mol}^{-1}$

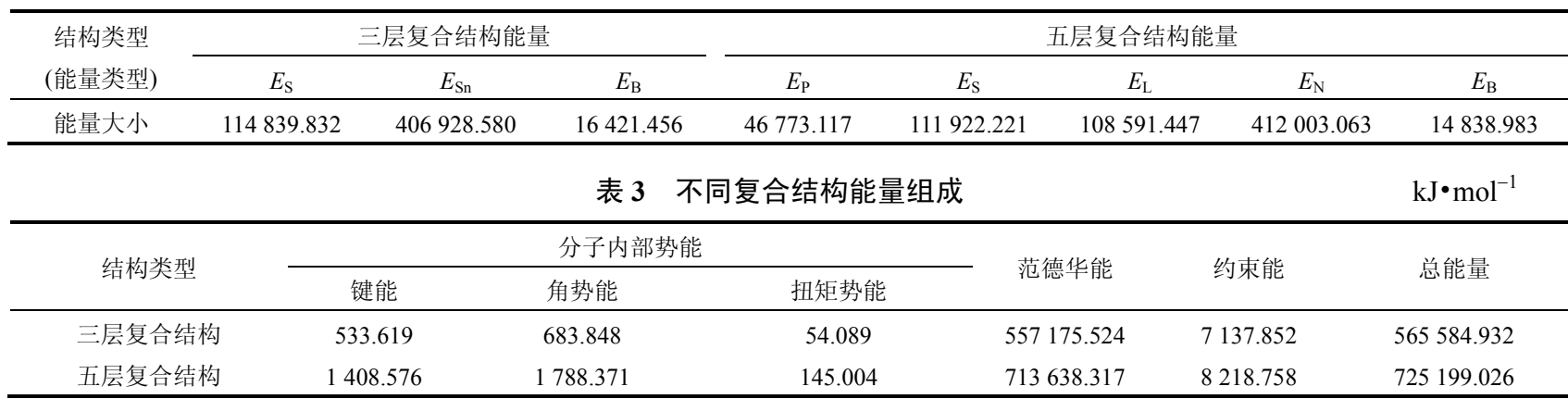

计算结果显示，不同结合层数的祄套界面结合 主要的作用力为范德华力和静电力, 界面间的相互 作用能主要为范德华能和静电能, 约束能相对比较 小, 可以忽略。影响界面结合能的主要因素为非键 结能。

\section{2 相邻材料间界面结合能}

油膜轴承在工作过程中, 复合材料祄套由于受 到压力载荷的作用容易发生片状脱落, 其根本原因 很大程度是衬套各层材料相邻界面间的结合性能不 足 $^{[28]}$ 。接下来从分子层面对比研究不同结合层数结 构祄套相邻界面间的结合能, 并以此判断复合材料 衬套的危险结合界面。

对结合界面相邻两材料中各部分材料的能量进
行计算。计算相邻材料界面结合能时，去除多余层 材料, 保留结合界面处相邻的两层材料, 定义下层 材料为第一层材料, 上层材料为第二层材料, 利用 分子动力学计算结构整体的能量以及各层材料的能 量。三层结构与五层结构相邻两层材料结合时整体 的能量以及各层材料的能量, 见表 4、5。综上可知, 对比不同复合结构衬套相邻合金界面结合能如表 6 所示。

表 4 三层复合结构衬套相邻界面结构能量 $\mathrm{kJ} \cdot \mathrm{mol}^{-1}$

\begin{tabular}{lcc}
\hline \multicolumn{1}{c}{ 相邻界面类别 } & 钢体层-镀锡层 & 镀锡层-巴氏合金层 \\
\hline 总能量 $E_{\mathrm{T}}$ & 2290839.616 & 424465.787 \\
第一层材料能量 $E_{1}$ & 114839.832 & 406928.580 \\
第二层材料能量 $E_{2}$ & 406928.580 & 16421.456 \\
界面结合能 & 26279.342 & 1115.751 \\
\hline
\end{tabular}

表 5 五层复合结构衬套相邻界面结构能量

$\mathrm{kJ} \cdot \mathrm{mol}^{-1}$

\begin{tabular}{lccc}
\hline \multicolumn{1}{c}{ 相邻界面类别 } & 防护层-钢体 & 钢体-钢铅合金 & 钢铅合金-镍栅层 \\
\hline 总能量 $E_{\mathrm{T}}$ & 39664.367 & 231120.639 & 532462.361 \\
第一层材料能量 $E_{1}$ & 46773.117 & 111922.221 & 108591.447 \\
第二层材料能量 $E_{2}$ & 111922.221 & 108591.447 & 412003.063 \\
界面结合能 & 7101.716 & 10606.972 & 11867.852 \\
\hline
\end{tabular}


表 6 三层与五层复合结构衬套相邻合金界面结合能

$\mathrm{kJ} \cdot \mathrm{mol}^{-1}$

\begin{tabular}{|c|c|c|c|c|c|c|}
\hline 结构类型 & \multicolumn{2}{|c|}{ 三层复合结构衬套 } & \multicolumn{4}{|c|}{ 五层复合结构祄套 } \\
\hline 相邻界面类别 & 钢体-镀锡层 & 镀锡层-巴氏合金 & 防护层-钢体 & 钢体-钢铅合金 & 钢铅合金-镍栅层 & 镍栅层-巴氏合金 \\
\hline 界面结合能 & 26279.342 & 1115.751 & 7101.716 & 10606.972 & 11867.852 & 1493.660 \\
\hline
\end{tabular}

为了更直观地比较相邻界面之间的能量, 如图 6 所示。

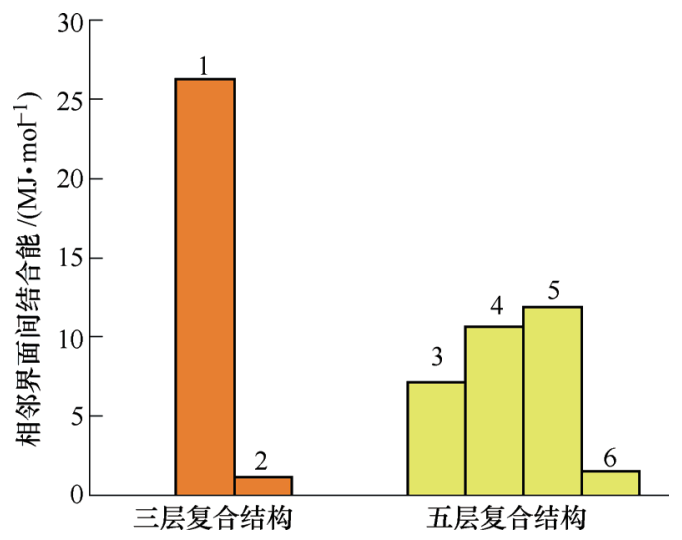

图 6 不同层数结构衬套相邻合金界面的结合能

（三层复合结构：1. 钢体一镀锡层 2. 镀锡层一巴氏合金层; 五层复合结构 3. 防护层一钢体层 4. 钢体一钢铅合金层 5. 钢铅合金一镍栅层 6. 镍栅层一巴氏合金层)

对比分析相邻两合金之间的界面结合能发现， 五层复合结构祄套中，钢铅合金层与镍栅层之间的 界面结合能最大, 达到了 $11867.852 \mathrm{~kJ} \cdot \mathrm{mol}^{-1}$; 镍 栅层与巴氏合金层的界面结合能最小，为 1493.660 $\mathrm{kJ} \cdot \mathrm{mol}^{-1}$ 。在三层复合结构祄套中, 镀锡层与巴氏 合金之间的结合能最小, 为 $1115.751 \mathrm{~kJ} \cdot \mathrm{mol}^{-1}$ : 从 表 6 可以得出, 结合界面的层数对结合性能有着明 显的影响, 五层复合结构祄套中最小界面结合能比 三层复合结构祄套最小界面结合能大 $33.87 \%$ 。由此 说明在工况条件下，五层复合结构祄套中，钢铅合 金与镍栅层之间粘接最牢固, 镍梛层与巴氏合金层 之间的粘接性能最差，最容易发生脱落或者断裂; 三层复合结构祄套中, 最易发生脱落的是镀锡层与 巴氏合金层。从宏观层面分析, 工程应用中, 两种 金属材料结合形成的界面由于材料性质的差异, 以 及界面结合形状不同, 在界面处会产生较大的奇异 性应力, 该应力会对界面结合强度以及材料的本身 的强度都会造成较大的影响, 并且材料差异性越大, 则界面奇异应力越明显, 添加过渡材料能够有效降 低复合材料界面处的奇异性应力, 镀合金层能缓和 由于成分突变而引起的应力集中, 有利于减少界面 物理性能的突变。对比分析不同复合结构祄套, 由 于五层复合结构每层材料之间材料性能逐渐过渡, 相比较三层复合结构祄套, 五层复合结构相邻材料 间的差异性较小, 能够有效降低结合界面处的奇异
应力, 从而提高界面结合性能。该结论与宏观规律 相一致。

\section{3 结论}

（1）根据巴氏合金中 $\mathrm{Cu}_{6} \mathrm{Sn}_{5} 、 \mathrm{SnSb} 、 \mathrm{Sn}$ 三种组 分含量配比建模, 通过对比两种结构祄套结合界面 的最小结合能可知, 结合界面的层数对结合性能有 着明显的影响，五层复合结构祄套结合界面结合性 能优于三层复合结构祄套的结合界面。五层复合结 构祄套中最小界面结合能比三层复合结构祄套最小 界面结合能大 $33.87 \%$ 。

(2) 从不同界面能量组成可以看出, 不同结合 层结构的祄套结合界面中主要的作用力为范德华力 和约束力, 可见范德华能和约束能在界面间的相互 作用中起主要作用, 分子内部势能比较小可以忽略。 影响界面结合能的主要因素为非键结能。

（3）通过对比分析祄套相邻合金界面间的结合 能发现，不同复合层结构的祄套导致的危险结合界 面不同。三层复合结构祄套中，最易发生脱落的界 面是镀锡层与巴氏合金层。五层复合结构祄套中， 钢铅合金层与镍栅层之间结合性能最好，粘接最牢 固; 镍栅层与巴氏合金层间结合性能最差, 最易发 生脱落失效。

\section{参 考 文 献}

[1] 刘晓涛, 张廷安. 层状金属复合材料生产工艺及其新进 展[J]. 材料导报, 2002, 16(7): 41-43.

LIU Xiaotao, ZHANG Tingan. Production process and new progress of layered metal composites[J]. Materials Guide, 2002, 16(7): 41-43.

[2] 田广民，李选明，赵永庆，等. 层状金属复合材料加工 技术研究现状[J]. 中国材料进展, 2013(11): 696-701. TIAN Guangmin, LI Xuanming, ZHAO Yongqing, et al. Research status of laminated metal composites machining technology[J]. Progress in Chinese Materials, 2013(11): 696-701.

[3] 王建梅, 薛亚文, 侯成, 等. 巴氏合金 ZChSnSb11-6 的蠕变特性 [J]. 材料科学与工程学报, 2013, 31(6): 785-789.

WANG Jianmei, XUE Yawen, HOU Cheng, et al. Creep properties of ZChSnSb11-6 alloy[J]. Journal of Materials 
Science and Engineering，2013，31(6): 785-789.

[4] 王建梅, 薛亚文, 蔡敏. 油膜轴承巴氏合金 ZChSnSb11-6 祄套的蠕变寿命评估 [J]. 上海交通大学 学报, 2015, 49(1): 62-66.

WANG Jianmei, XUE Yawen, CAI Min. Creep life evaluation of ZChSnSb11-6 bushing of oil film bearing[J]. Journal of Shanghai Jiaotong University, 2015, 49(1): 62-66.

[5] WANG Jianmei, MENG Fanning, ZHANG Xiaotian, et al. Mathematical model and algorithm of interface singular stress field of oil-film bearing[J]. Tribology International, 2017(116): 351-361.

[6] 黄庆学, 王建梅, 静大海, 等. 油膜轴承雉套过盈装配 过程中的压力分布及损伤 [J]. 机械工程学报, 2006, 42(10): 102-108.

HUANG Qingxue, WANG Jianmei, JING Dahai, et al. Pressure distribution and damage during interference fitting of oil film bearing cone sleeve[J]. Journal of Mechanical Engineering, 2006, 42 (10): 102-108.

[7] 黄庆学, 李璞, 王建梅, 等. 宏微观跨尺度下的雉套运 行力学机理研究 $[J]$. 机械工程学报, 2016, 52(14): 213-220

HUANG Qingxue, LI Pu, WANG Jianmei, et al. A study on the mechanism of operation of cone sleeve in macro and micro scale[J]. Journal of Mechanical Engineering, 2016, 52(14): 213-220.

[8] 王晓虹, 谷晓燕, 孙大千. 钢/铝异种金属激光焊接头 界面特性的研究[J]. 机械工程学报, 2016, 53(4): 26-33. WANG Xiaohong, GU Xiaoyan, SUN Daqian. Study on interface characteristics of laser welded joints of steel/aluminum dissimilar metals[J]. Journal of Mechanical Engineering, 2016，53(4): 26-33.

[9] 杜波, 孙转平, 杨新岐, 等. 异种铝合金摩擦塞补焊接 头微观组织及性能 [J]. 机械工程学报, 2017, 53(4): 43-48.

DU Bo, SUN Zhuanping, YANG Xinqi, et al. Microstructure and properties of dissimilar aluminum alloy friction plug Joint[J]. Journal of Mechanical Engineering, 2017, 53 (4): 43-48.

[10] LIU Xuefei, LIU Hanlian, HUANG Chuanzhen, et al. Synergistically toughening effect of $\mathrm{SiC}$ whiskers and nanoparticles in $\mathrm{Al}_{2} \mathrm{O}_{3}$-based composite ceramic cutting tool material[J]. Chinese Journal of Mechanical Engineering, 2016, 29(5): 977-982.

[11] LUO Mengzi, LIANG Li, LANG Lin, et al. Molecular dynamics simulations of the characteristics of $\mathrm{Mo} / \mathrm{Ti}$ interfaces[J]. Computational Materials Science, 2018, 141: 293-301.

[12] CHEN Yujie, ZOU Yu, SUN Dongliang, et al. Molecular dynamics simulation of bubble nucleation on nanostructure surface $[\mathrm{J}]$. International Journal of Heat \& Mass Transfer, 2018, 118: 1143-1151.

[13] BEJAUD R, DURINCK J, BROCHARD S. Twininterface interactions in nanostructured $\mathrm{Cu} / \mathrm{Ag}$ : Molecular dynamics study[J]. ActaMaterialia, 2018, 144: 314-324.

[14] ADJAOUD O, ALBE K. Microstructure formation of metallic nanoglasses: Insights from molecular dynamics simulations[J]. ActaMaterialia, 2018, 145: 322-330.

[15] WU Chengda, HOU Chunjen. Molecular dynamics analysis of plastic deformation and mechanics of imprinted metallic glass films[J]. Computational Materials Science, 2018, 144: 248-255.

[16] LASHGARI H R, TANG C, CHU D, et al. Molecular dynamics simulation of cyclic indentation in Fe-based amorphous alloy[J]. Computational Materials Science, 2018, 143: 473-479.

[17] MOJUMDER S, DATTA D. Molecular dynamics study of plasticity in $\mathrm{Al}-\mathrm{Cu}$ alloy nanopillar due to compressive loading[J]. Physica B Condensed Matter, 2017, 530: 86-89.

[18] SARKAR J, DAS D K. Nanoindentation study of mechanical behavior and response of a single layer pristine silicene sheet using molecular dynamics simulations[J]. Computational Materials Science, 2018, 147: $64-71$

[19] WANG Jianmei, MENG Fanning, LI Zhixiong, et al. Research on interface bonding energy of multi-layer model on ZChSnSb /FeSn2/Steel[J]. Tribology International, 2018(123): 37-42.

[20] 廖瑞金, 朱孟兆, 周欣, 等. 油纸复合介质中水分子扩 散行为的分子动力学模拟 [J]. 物理化学学报, 2011 , 27(4): 815-824.

LIAO Ruijin, ZHU Mengzhao, ZHOU Xin, et al. Molecular dynamics simulation of water diffusion in oil-paper composite media[J]. Journal of Physicochemistry, 2011, 27 (4): 815-824.

[21] BECTON M, ZHANG L, WANG X. On the crumpling of polycrystalline graphene by molecular dynamics simulation.[J]. Physical Chemistry Chemical Physics, 2015, 17(9): 6297-304.

[22] SUN Chengzhen, BAI Bofeng. Molecular sieving through a graphenenanopore: non-equilibrium molecular dynamics simulation[J]. Science Bulletin, 2017, 62(8): 554-562.

[23] SEIFOORI S, HAJABDOLLAHI H. Impact behavior of single-layered graphene sheets based on analytical model and molecular dynamics simulation[J]. Applied Surface Science, 2015, 351(12-13): 565-572.

[24] 李健, 杨延清, 罗贤, 等. 分子动力学模拟在复合材料 
界面研究中的进展 [J]. 稀有金属材料与工程, 2013, 42(3): 644-648.

LI Jian, YANG Yanqing, LUO Xian, et al. Progress of molecular dynamics simulation in the study of composite interface[J]. Rare Metal Materials and Engineering, 2013, $42(3): 644-648$.

[25] 杜立群, 郭照沛, 张晓蕾, 等. 交联 SU-8 光刻胶与 Ni 基底结合性的分子动力学模拟 [J]. 高分子学报, 2010(6): 629-634.

DU Liqun, GUO Zhaopei, ZHANG Xiaolei, et al. Molecular dynamics simulation of the binding of crosslinked SU-8 photoresist to Ni substrate[J]. Journal of Polymer, 2010 (6): 629-634.

[26] 王尧, 王建梅, 黄玉琴, 等. 锡界面层最佳厚度试验与 模拟研究[J]. 机械工程学报, 2015, 51(20): 106-113.
WANG Yao, WANG Jianmei, HUANG Yuqin, et al. Experimental and simulation study on the optimum thickness of tin interfacial layer[J]. Journal of Mechanical Engineering, 2015, 51(20): 106-113.

[27] LI Shaofan, URATA Shingo. An atomistic-to-continuum molecular dynamics : Theory, algorithm , and applications[J]. Computer Methods in Applied Mechanics \& Engineering, 2016, 306: 452-478.

[28] WANG Jianmei, XIA Quanzhi, MA Yang, et al. Interfacial bonding energy on the interface between $\mathrm{ZChSnSb} / \mathrm{Sn}$ alloy layer and steel body at microscale[J]. Materials, 2017, 10(10): 1128 .

作者简介: 王建梅(通信作者), 女, 1972 年出生, 博士，教授。主要研 究方向为工业摩擦学和界面科学。

E-mail: wjmhdb@163.com 